\title{
Meta
}

Journal des traducteurs

Translators' Journal

\section{STOLZE, R. (2003) : Hermeneutik und Translation, Tübinger Beiträge zur Linguistik 467, Tülbingen, Gunter Narr, 348 p.}

\section{Bernd Stefanink}

Volume 49, numéro 4, décembre 2004

URI : https://id.erudit.org/iderudit/009795ar

DOI : https://doi.org/10.7202/009795ar

Aller au sommaire du numéro

Éditeur(s)

Les Presses de l'Université de Montréal

ISSN

0026-0452 (imprimé)

1492-1421 (numérique)

Découvrir la revue

Citer ce compte rendu

Stefanink, B. (2004). Compte rendu de [STOLZE, R. (2003) : Hermeneutik und Translation, Tübinger Beiträge zur Linguistik 467, Tübingen, Gunter Narr, 348 p.] Meta, 49(4), 938-941. https://doi.org/10.7202/009795ar

Ce document est protégé par la loi sur le droit d'auteur. L'utilisation des services d'Érudit (y compris la reproduction) est assujettie à sa politique d'utilisation que vous pouvez consulter en ligne.

https://apropos.erudit.org/fr/usagers/politique-dutilisation/
Cet article est diffusé et préservé par Érudit.

Érudit est un consortium interuniversitaire sans but lucratif composé de l’Université de Montréal, l'Université Laval et l'Université du Québec à Montréal. Il a pour mission la promotion et la valorisation de la recherche. https://www.erudit.org/fr/ 
de noblesse théoriques. On y retrouve à mon avis le modèle tripartite de Nida - analyse, transfert, restructuration - publié quatre ans auparavant ${ }^{3}$ et une volonté d'asseoir l'interprétation dans le giron universitaire.

La relation entre sciences cognitives et interprétation est illustrée par plusieurs modèles: le modèle de Massaro, le modèle de Moser et le modèle de répartition d'efforts de Gile. Les recherches en neuropsychologie et en neurolinguistique se focalisent notamment sur le bilinguisme et le rôle de la mémoire en interprétation simultanée. De même, l'auteur prend en considération les tests d'aptitude à l'interprétation ainsi que les exercices de propédeutique, pour conclure avec les développements les plus récents en la matière et la mise au point de critères de qualité satisfaisants au plan méthodologique.

C'est précisément ce dernier point, la qualité, problème encore entier, qui est au cœur du cinquième et dernier chapitre, Qualità. La qualité est à mon sentiment la question cruciale de l'interprétation aujourd'hui et la définition de cette notion a des implications évidentes tant sur la formation que sur la pédagogie du marché. Il est vrai, comme l'affirmait Seleskovitch, qu' «un interprète médiocre ne sert à rien». En l'absence de critères de qualité qui fassent l'unanimité, je me range volontiers à l'opinion de Gentile: «invariably and inexorably the tendency will be to concentrate on the evaluation of language skills, in part because it is easier to justify one's assessment in terms of language than in terms of communication ${ }^{4} »$.

L'analyse des critères de qualité se fonde sur des variables comme le respect du contenu et la fonction de communication, et fait le départ entre l'interprétation directe pour le public et le relais qui obéit évidemment à d'autres impératifs. Au bout devrait se trouver la notion d'équivalence, ce casse-tête traductologique qui ne cesse de hanter les esprits depuis de nombreux siècles. Bien entendu, l'ambition de l'auteur n'est pas de résoudre le problème, mais de baliser les acquis récents et d'ouvrir des pistes prometteuses. J'aurais néanmoins souhaité que la réalité quotidienne du marché soit plus longuement évoquée.

Le livre s'achève par une bibliographie très abondante et particulièrement fouillée. Le lecteur candide comme l'interprète professionnel y trouveront nombre de références intéressantes et un point de départ utile pour d'éventuelles recherches.

Christian Balliu

Haute École de Bruxelles, Bruxelles, Belgique

\section{NOTES}

1. Voir Jean Herbert: «How conference interpretation grew», in David Gerver and H. Sinaiko (éd.), Language Interpretation and Communication, New York/Londres, Plenum Press, 1978, p. 6 et suivantes.

2. Il y a heureusement des exceptions, par exemple: les Mémoires d'Eugen Dollmann (1963), l'interprète des tête-à-tête entre Hitler et Mussolini, ou le Silent Missions de Vernon Walters (1978), l'interprète de Truman, Eisenhower, Nixon et Kissinger.

3. Eugene A. Nida, Toward a Science of Translating, Leyde, E.-J. Brill, 1964.

4. Adolfo Gentile, «Community interpreting or not?», in Silvana E. Carr et al. (éd.), The Critical Link, Interpreters in the Community, Amsterdam/Philadelphia, John Benjamins, 1997, p. 116.

Stolze, R. (2003): Hermeneutik und Translation, Tübinger Beiträge zur Linguistik 467, Tübingen, Gunter Narr, 348 p.

Cette dernière somme de la pensée herméneutique en traduction, vient s'inscrire dans la ligne de pensée de Stolze, telle qu'elle se manifeste dans de nombreux articles et de façon plus compacte dans Hermeneutisches Übersetzen, également parue chez Narr, en 1992. Onze ans plus tard, Stolze récidive. La similitude des titres pourrait laisser penser que l'on assiste à une réédition plus ou moins corrigée et augmentée. Erreur ! 
Dans cette dernière version de sa pensée, Stolze insiste sur les bases philosophiques de sa conception de la traduction, qui trouve son origine dans la philosophie de Heidegger et de Gadamer. Heidegger lui a montré que le «cercle vicieux» des herméneutes, n'a en fait rien de vicieux, qu'il faut, au contraire, y voir une possibilité d'approcher le sens dans ce qu'il a de plus originel. Ceci sans toutefois avoir la prétention de découvrir la vérité du texte et, encore moins, l' «intention de l'auteur», comme elle reproche à l'approche intrprétative de Lederer (1994) de vouloir le faire ${ }^{1}$. Non, le traducteur ne doit même pas prétendre traduire le (!) sens du texte. Le traducteur traduit ce qu'il comprend. Le texte recèle, en effet, un Sinnüberschuss (c'est-à-dire un «trop de sens» ou «surplus de sens»). Et ce sens n'est pas dans le texte, mais jaillit de la mise en contact du récepteur avec le texte, dans ce que Gadamer a appelé la «Horizontverschmelzung» («fusion des horizons»), c'est-à-dire une empathie (Stolze n'utilise toutefois pas ce terme) avec le texte, dans laquelle le récepteur du texte se fond dans le texte pour participer au Wahrheitsgeschehen, c'est-à-dire à la vérité (dynamique) du texte. Un sens à chaque fois différent, en fonction des vécus des différents récepteurs, ou, s'il s'agit d'un seul récepteur, en fonction de ce que l'on pourrait appeler, en termes cognitivistes, de l'«être-en-situation» (la Situiertheit de Rickheit et Strohner 1993) du récepteur au moment de sa réception.

Cette Horizontverschmelzung du traducteur/récepteur avec le texte est si intense que le traducteur est saisi $i^{2}$ d'une «impulsion de formulation intuitive» intuitiver Formulierungsimpuls, dans un "processus autopoïétique en partie inconscient» (partiell unbewussten autopoietischen geistigen Prozess), qui fait passer le texte de l' «enveloppe» («Hülle») de la LS vers celle de la LC, de sorte qu'on ne doit pas voir l'opération traduisante comme un Entscheidungsprozess ${ }^{3}$ (un "processus de prise de décision»), ni le traducteur comme se trouvant «entre» le texte TS et le TC. L'image du traducteur/passeur, qui fait passer le sens/ bac d'une rive à l'autre - comme nous l'avons encore dans le titre du volume consacré aux 70 ans de Katharina Reiß - est out!

Cette priorité de l'approche intuitive implique aussi de nouvelles méthodes d'évaluation. Stolze parle de la nécessité de rendre plausible à autrui et parle d'une Intersubjektive Überprüfbarkeit, c'est-à-dire d'une «vérifiabilité intersubjective». Dans Stefanink (1997), où nous exposons des idées du même ordre, en parlant de cette priorité accordée à l'intuition comme d'une «epistemologische Wende» (un «bouleversement épistémologique») nous avons introduit le terme de Intersubjektive Nachvollziehbarkeit, qui nous semble préférable, dans la mesure où Nachvollziehbarkeit implique une certaine dynamique de la part du sujet évaluant, qui doit suivre la dynamique du cheminement intuitif/associatif de la pensée du traducteur vers une solution créative. Par ailleurs Nachvollziehbarkeit focalise l'attention sur le sujet évaluant, alors que Überprüfbarkeit est centré sur l'objet à évaluer. Il nous semble que Nachvollziehbarkeit s'intègre plus radicalement dans le cadre d'une herméneutique qui met l'accent sur le facteur humain et le côté dynamique de la constitution du sens. Dans le français «plausibilité interindividuelle» qui serait la traduction la plus proche pour Intersubjektive Nachvollziehbarkeit cette connotation de dynamisme se perd évidemment.

Pour le reste, n'importe quel traducteur chevronné ne pourra que souscrire à la description Stolzienne du processus de traduction. Si l'on a pu reprocher un certain mysticisme à l'approche herméneutique, il n'en est pas moins vrai que c'est bien ainsi que le traducteur approche le texte, loin des exercices de dissection du texte, préconisés par Heidrun Gerzymisch-Arbogast et Klaus Mudersbach (1998), pour lesquels ces derniers s'arrogent le label exclusif de méthode «scientifique $»^{4}$.

Si l'on veut une justification «scientifique» de l'approche herméneutique qui, il est vrai, reste quelque peu cantonnée dans l'heuristique, on la trouvera plutôt dans un faisceau fascinant de recherches, telles qu'elles sont menées à l'heure actuelle, dans les disciplines parallèles, par des linguistes cognitivistes comme Lakoff ou Langacker, par des chercheurs en mémoire comme Schank, ou encore par des chercheurs en créativité comme de Bono ou 
Guilford (avec la restriction que Guilford considère la créativité comme une problem solving activity, ce qui, là encore, va à l'encontre de l'exclusion du Entscheidungsprozess de l'opération traduisante, à laquelle procède Stolze, comme nous l'avons vu et si nous avons bien compris $^{5}$ ).

Stolze s'étonne que les cognitivistes n'aient pas mieux tenu compte de l'approche herméneutique, puisque «In jüngster Zeit erbringt nämlich die kognitive Forschung vielfach Ergebnisse, welche die älteren Behauptungen der Hermeneutik bestätigen» (p. 38) (= les résultats récents des recherches cognitivistes viennent, en effet, confirmer sur bien des points les affirmations plus anciennes de l'herméneutique). Elle trouve toutefois des différences entre ces deux approches, dans la mesure où les cognitivistes conçoivent la «gestion du texte» comme une "construction du sens», alors que pour l'herméneutique il s'agit plutôt d'une «expérience du sens». Les cognitivistes voient l'accès au sens comme une Textverarbeitung donc un processus actif de construction du sens, alors que l'herméneute attend que le texte "l'interpelle», "s'adresse à lui» (mich anspricht) (p. 91). À la page 195 elle semble pourtant se contredire (?) et considérer le récepteur du texte comme participant activement à la constitution du sens: «Damit appellieren die Wörter aber an die Mitarbeit des Lesers» (=Ainsi les mots font appel à/sollicitent la participation active du lecteur).

Un livre qui vaut la peine d'être lu avec la Achtsamkeit, recommandée par Brodbeck pour toute découverte créative.

Bernd Stefanink

Université de Bielefeld, Bielefeld, Allemagne

\section{NOTES}

1. Sans toutefois tenir compte de la nuance fine introduite par Lederer entre «intention de l'auteur» et "vouloir dire de l'auteur»; en fait c'est la prétention de vouloir retrouver le «vouloir dire de l'auteur » que fustige Stolze, même si elle parle de l' «intention de l'auteur»; Lederer, en effet, définit "l'intention de l'auteur» comme les sous-entendus implicites par lesquels on veut exercer une influence sur le comportement du récepteur, par ex. : l'inciter à cesser de fumer en disant que quelqu'un d'autre a cessé de fumer et en sous-entendant que le récepteur du texte pourrait en faire autant. Traduire cette intention serait faire de l'exégèse, que Lederer condamne, la distinguant de l'interprétation, qui est à la base de l'approche interprétative.

2. N.B. : Stolze utilise le terme de Überwältigtsein du traducteur par la vérité du texte. Ce mot qui, selon les contextes, peut signifier "être vaincu», «être subjugé» a la même racine que Vergewaltigung qui veut dire "viol», et on pourrait dire que le traducteur est en quelque sorte violé par la vérité du TS, à l'inverse de l'image du viol utilisé par Jean-René Ladmiral (1993) pour qui c’est le traducteur qui viole le texte source.

3. Sur ce point nous avons quelque mal à suivre la pensée de Stolze. Comment peut-on exclure le Entscheidungsprozess de l'opération traduisante? Les résultats de nos recherches empiriques, qui consistent en des analyses procédurales de corpus que nous avons recueillis par les méthodes de l'analyse conversationnelle ethnométhodologique, montrent à l'évidence et de façon nettement matérialisée des phases où les traducteurs se trouvent devant des choix traduisants à opérer, même si l'opération traduisante dans son ensemble ne peut, certes, être considérée comme un continuel decision making, comme d'aucuns le proclament.

4. «Intuitives Erfassen ist methodischem Untersuchen vorgeordnet» (p. 108) (="La saisie intuitive du texte précède l'analyse méthodique). Elle s'oppose ainsi résolument aux affirmations contraires de pour qui l'intuition a sa chance, à partir du moment où la pensée logique est à bout de ressources.

5. Cf. Balacescu/Stefanink 2003.

\section{RÉFÉRENCES}

Balacescu, I. et B. STEFAnink (2003) : «Modèles explicatifs de la créativité en traduction», Meta 48-4, p. 509-525.

Gerzymisch-Arbogast, H. und K. Mudersbach (1998): Methoden des wissenschaftlichen Übersetzens, Tübingen, Francke. 
Ladmiral, J.-R. (1993): "Sourciers et ciblistes» dans Holz-Mänttäri, J. und C. Nord: Traducere Navem. Festschrift für Katharina Reiß, Tampere, Studia Translatologica, ser. A vol. 3. Lederer, M. (1994): La traduction aujourd'hui, Paris, Hachette.

Rickheit, G. und H. Strohner (1993): Grundlagen der kognitiven Sprachverarbeitung, Modelle, Methoden, Ergebnisse, Tübingen, Francke.

Stefanink, B. (1997) : «Esprit de finesse - Esprit de géométrie: Das Verhältnis von Intuition und übersetzerrelevanter Textanalyse" beim Übersetzen», dans R. Keller (Hrsg.) (1997): Linguistik und Literaturübersetzen, Tübingen, Narr, p. 161-183.

Wilss, W. (1988): Kognition und Übersetzen. Zu theorie und Praxis der menschlichen und maschinellen Übersetzung, Tübingen, Niemeyer.

\section{MA, H. J. (2003): A Study on Nida's Translation Theory, Beijing, Foreign Language Teaching and Research Press, $250 \mathrm{p}$.}

Eugene A. Nida (1914-) is a distinguished American translation theorist as well as linguist. His translation theory has exerted a tremendous influence on translation studies in western countries. His works on translation set off the study of modern translation as an academic field (Snell-Hornby 1988:1; Heylen 19993:4; Baker 1998:277), and he is regarded as the most influential one among all contemporary translation theorists (New Mark 1993:133).

Nida's influence upon Chinese translation studies is greater still. Before his theory was introduced into China in the 1980s, scholars mainly focused attention on traditional Chinese translation theories, especially Yan Fu's (1854-1921) three-character principle of translation, i.e. faithfulness, smoothness and elegance. Since Nida's theory was grounded solidly on contemporary developments of linguistics, communication theory, information theory, semiotics and anthropology, Chinese translation scholars took great interest in his theory. In fact, Nida's and his theory have been a hot topic in China for almost two decades. In the beginning, Nida's theory was extensively introduced and much discussed among translation scholars, and was given a high profile for its new perspectives on translation. As time went by, his theory was questioned and challenged. In recent years, his theory was strongly criticized, and even considered as outdated and having outlived its usefulness.

It is evident that Nida's translation theory has undergone dramatic ups and downs since it was introduced into China. This phenomenon is very interesting and has provoked a series of reflections on the part of the Chinese translation scholars. Why is there such a big change in Chinese translation scholars' attitudes towards Nida's theory? Are the criticisms against his theory justified? What are the demerits for which his theory is being severely criticized? Is his theory really useless or outdated for Chinese translation studies, as some scholars have so argued?

All these questions about Nida's theory are of great significance in the sense that, to some degree, they determine how Nida's theory will be received in the future Chinese translation studies. Something cautions us against taking leave of his theory without further consideration.

A Study on Nida's Translation Theory is the first attempt to make a systematic study on Nida's translation theory in the Chinese translation community. It reviews the reception of Nida's theory both in China and in the western countries from a historical perspective, explores its important concepts, such as his "science of translation," "dynamic equivalence"/ "functional equivalence," and the decoder's channel capacity. It also tries to clarify misunderstandings and misconceptions concerning his theory, including his theory of "reader's response," his advocacy of naturalizing translation, and the relationship between his "science of translation" and Chomsky's transformational generative grammar. Examples from Today's English Version and Today's Chinese Version of the Bible convincingly prove that, 